\title{
Neuroimaging Reveals Distinct Brain Glucose Metabolism Patterns Associated with Morphine Consumption in Two Inbreed Rat Models
}

\author{
$M^{\mathrm{a}}$ Luisa Soto-Montenegro ( $\square$ marisa@hggm.es) \\ Instituto de Investigación Sanitaria Gregorio Marañón \\ Verónica García \\ Instituto de Investigación Sanitaria Gregorio Marañón \\ Nicolás Lamanna-Rama \\ Instituto de Investigación Sanitaria Gregorio Marañón \\ Gonzalo López-Montoya \\ Instituto de Investigación Sanitaria Gregorio Marañón \\ Manuel Desco \\ Universidad Carlos III de Madrid \\ Emilio Ambrosio \\ Universidad de Educación Nacional a Distancia (UNED)
}

\section{Research Article}

Keywords: morphine self-administration, FDG-PET, glucose metabolism, drug addiction, Lewis and Fischer strains

Posted Date: May 5th, 2021

DOI: https://doi.org/10.21203/rs.3.rs-472435/v1

License: (9) This work is licensed under a Creative Commons Attribution 4.0 International License. Read Full License 
Regular Research Article

\section{Neuroimaging reveals distinct brain glucose metabolism patterns} associated with morphine consumption in two inbreed rat models

$M^{\text {a }}$ Luisa Soto-Montenegro ${ }^{1,2, *}$, Verónica García ${ }^{1}$, Nicolás Lamanna-Rama ${ }^{1}$, Gonzalo López-Montoya ${ }^{1,4}$, Manuel Desco ${ }^{3,2,1,5}$, Emilio Ambrosio ${ }^{4, *}$.

${ }^{1}$ Instituto de Investigación Sanitaria Gregorio Marañón, Madrid, Spain.

${ }^{2}$ CIBER de Salud Mental (CIBERSAM), Madrid, Spain.

${ }^{3}$ Departamento de Bioingeniería e Ingeniería Aeroespacial, Universidad Carlos III de Madrid, Spain.

${ }^{4}$ Departamento de Psicobiología, Facultad de Psicología, Universidad de Educación Nacional a Distancia (UNED), Madrid, Spain.

${ }^{5}$ Centro Nacional de Investigaciones Cardiovasculares, CNIC, Madrid, Spain

${ }^{*}$ Corresponding authors

Running title: Rat strain differences in brain ${ }^{18}$ F-FDG-PET: implication in morphine abuse

\section{Corresponding authors:}

Mํㅡㄴ Luisa Soto Montenegro; Laboratorio de Imagen. Medicina Experimental. Hospital General Universitario Gregorio Marañón. Dr. Esquerdo, 46. E-28007 Madrid. Spain. e-mail: marisa@hggm.es

Emilio Ambrosio; Departamento de Psicobiología, Facultad de Psicología, Universidad de Educación Nacional a Distancia (UNED), Madrid, Spain. e-mail: eambrosio@psi.uned.es 


\section{ABSTRACT}

Addiction vulnerability is the individual's risk of developing an addiction during their lifetime. A challenge in the neurobiology of drug addiction is understanding why some people become addicted to drugs. Here, we used positron emission tomography (PET) and statistical parametric mapping (SPM) to evaluate changes in brain glucose metabolism in response to chronic morphine self-administration (MSA) in two rat strains with different vulnerability to drug abuse, Lewis (LEW) and Fischer 344 (F344). Four groups of animals were trained to self-administer morphine or saline for 15 days. 2-deoxy-2- $\left[{ }^{18} \mathrm{~F}\right]-$ fluoroD-glucose (FDG)-PET studies were performed on the last day of MSA (acquisition phase) and after 15 days of withdrawal. PET data were analyzed using SPM12. LEW-animals self-administered more morphine injections per session than F344-animals. We found significant brain metabolic differences between LEW and F344 strains in the cortex, hypothalamus, brainstem, and cerebellum. In addition, the different brain metabolic patterns observed after the MSA study between these rat strains indicate differences in the efficiency of neural substrates to translate the drug effects, which could explain the differences in predisposition to morphine abuse between one individual and another. These findings have important implications for the use of these rat strains in translational morphine and opiate research.

KEYWORDS: morphine self-administration, FDG-PET, glucose metabolism, drug addiction, Lewis and Fischer strains 


\section{INTRODUCTION}

Addiction vulnerability is the individual's risk of developing an addiction during their lifetime. Clinical and preclinical studies have shown that among the biological factors involved in this disorder, genetic variability plays an important role in humans ${ }^{1-4}$, accounting for at least $40-60 \%$ of the variation in vulnerability to drug dependence ${ }^{5}$. In this regard, the use of inbred rats has proven to be a valuable tool for identifying differences in vulnerability to drug addiction. The Lewis (LEW) and Fischer 344 (F344) inbred rat strains have been the most widely used in modeling genetic vulnerability to drugs. LEW and F344 rats differ with respect to drug self-administration. Consequently, LEW rats more readily self-administer drugs, such as alcohol, opiates, and cocaine, than F344 rats ${ }^{6-13}$. At the neurochemical level, F344 rats have high basal levels of tyrosine hydroxylase $(\mathrm{TH})$ protein, proenkephalin, dopamine transporter, glutamate, and $\mu$-opioid receptors in the nucleus accumbens ${ }^{14,15}$. F344 rats also have high levels of $\mu$-opioid receptors in the striatum (both dorsal and ventral), lateral globus pallidus, basolateral and lateral amygdaloid nucleus, periaqueductal gray matter (PAG), substantia nigra (compact and reticular parts), and locus coeruleus measured by autoradiography ${ }^{11}$. LEW rats, on the other hand, have high basal levels of $\mathrm{TH}$ protein and dopamine D1 and NMDA receptors in the ventral tegmental area (VTA) ${ }^{12,16-18}$, as well as greater activity of the $\mu$-opioid receptor in the nucleus accumbens, septal nuclei, thalamus, VTA, raphe nuclei, and locus coeruleus ${ }^{11}$.

In line with these observations, functional neuroimaging techniques, such as positron emission tomography (PET), single-photon emission computed tomography, functional magnetic resonance imaging (fMRI), and electroencephalography, have been widely used to demonstrate the emotional and cognitive-behavioral components of addiction ${ }^{19,20}$, as well as to study the neurotoxic effects of drugs in the brain ${ }^{21-28}$. In this respect, PET with 2-deoxy-2$\left[{ }^{18} \mathrm{~F}\right]$-fluoro-D-glucose (FDG) has traditionally been the most commonly used technique for direct quantification of regional brain glucose metabolism in clinical and preclinical studies ${ }^{29-34}$. It constitutes an invaluable tool for investigating the in vivo changes in brain metabolism as a result of pharmacological manipulation. Given that the LEW and F344 strains show differential behaviours that model 
addiction and therefore different vulnerability to drugs, our working hypothesis is that the metabolism of morphine in reward areas of the brain would be higher in the LEW strain than in the F344 strain. As far as we know, this is the first study to examine the effects of morphine self-administration on glucose metabolism using in vivo FDG-PET and statistical parametric mapping (SPM) in two rat strains with different vulnerability to drug abuse.

\section{METHODS}

\section{$\underline{\text { Animals }}$}

Thirteen male F344 and 14 LEW inbred rats (250-300 g body weight) were obtained from the animal facility of the Universidad Nacional de Educación a Distancia (UNED). Animals were housed individually when they reached postnatal day 75 (PND75) in order to leave sufficient time between individual housing and the brain metabolic studies and thus avoid any non-specific effects of isolation stress on the metabolic measurements. All animal procedures were conducted in conformity with Directive 2010/63/EU of the European Parliament and of the Council, the ARRIVE guidelines, and approved by the Ethics Committee for Animal Experimentation of UNED and Hospital Gregorio Marañón (number ES280790000087).

\section{Drug Administration and Experimental Protocol}

Figure $1 \mathrm{~A}$ shows the drug treatment and the design of the study. Twelve operant chambers (Coulbourn Instruments, Allentown, PA, USA) were used for the operant food-reinforced behaviour and morphine self-administration studies. A lever designed to register a response to $3.0 \mathrm{~g}$ of force was placed on the front wall of the chamber ${ }^{11}$. Food and morphine operant data were acquired and stored on IBM computers (Med Associates, PA, USA).

Saline or morphine sulphate self-administration $(1 \mathrm{mg} / \mathrm{kg} / \mathrm{injection}$ in $12 \mathrm{~h}$ daily sessions) was studied with a fixed-ratio 1 (FR1) schedule of reinforcement for 15 days ${ }^{13}$.

Animals were divided into four groups based on the factors studied: rat strain (LEW and F344) and drug treatment (morphine and saline). The sample 
sizes of the groups were as follows: 1) LEW-saline $(n=6) ; 2)$ LEW-morphine ( $n$ $=8)$; 3) F344-saline ( $n=6)$; and 4) F344-morphine $(n=7)$.

\section{Fixed-ratio1 food-reinforced behavior}

This experimental procedure was performed at PND75. Before starting the training, animals were food deprived to $95 \%-90 \%$ of their free-feeding weight and submitted to an FR1 schedule of food reinforcement in which a single press of the lever turned on a light stimulus above the lever that signalled pellet delivery and reward availability (45 mg; Noyes Pellets, USA). Each delivery of a pellet was followed by a 30-second timeout period in which responses had no scheduled consequence (FR1: TO $30 \mathrm{~s}$ ). This food-reinforced behavior was acquired over 5 days (30 minutes each day). After this phase, the LEW and F344 rats had ad libitum access to food and were thus able to recover their free-feeding weight.

\section{Morphine self-administration}

When the response rate was sufficient (more than 50 responses per session) and stable (less than 10\% variation across three consecutive sessions), subjects were surgically prepared with a chronic intravenous catheter inserted into the right jugular vein. Polyvinylchloride tubing (0.064 i.d.) was implanted approximately at the level of the atrium under anesthesia with ketamine (40 $\mathrm{mg} / \mathrm{kg}$, i.p.) and diazepam (10 $\mathrm{mg} / \mathrm{kg}$, i.p.). The catheter was passed subcutaneously and located in the midscapular region; it then passed through a spring tether system (Alice King, Chatham, CA, USA) that was mounted on the skull of the rat with dental cement. The animals were given at least five days to recover from the surgery, and the catheters were flushed daily with $0.5 \mathrm{ml}$ of an antibiotic solution (gentamicin $40 \mathrm{mg} / \mathrm{ml}$ ) dissolved in heparinized saline to prevent infection and to maintain catheter patency.

The drug-reinforced behaviour study was performed during the dark cycle. It involved two phases: 1) The acquisition phase, which started at PND100 and consisted of sessions lasting 12 hours per day (starting at 8:00 pm) with morphine sulphate $(1 \mathrm{mg} / \mathrm{kg}$, dissolved in saline [0.9\% NaCl] solution) or saline alone under an FR1 schedule of reinforcement for 15 consecutive days; and 2) The withdrawal phase, where the drugs were discontinued for 15 consecutive days. 
During acquisition sessions, one active lever press resulted in morphine/saline infusion delivered over 10 seconds and followed by a 10-second time-out. A light cue located above the active lever indicated the availability of the drug; this was turned off only during drug delivery, time out, and at the end of each session. A limit of 50 infusions per session was set in order to avoid overdosing.

\section{FDG-PET Imaging Studies}

PET images were acquired on two separate days: the first session following morphine self-administration (MSA) (at the end of the MSA phase, -acquisition phase-) and the second session following withdrawal (at the end of the withdrawal phase).

Imaging was performed using a dedicated small animal PET scanner (rPET, SUINSA Medical Systems, Madrid). 2-deoxy-2-[18 F]fluoro-D-glucose (FDG: $1.99 \pm 0.24 \mathrm{mCi}$ ) was injected through the catheter inside the jugular vein, and, after an uptake period of 35 minutes, the animals were imaged for 60 minutes under isoflurane anesthesia $(5 \%$ for induction and $1-1.5 \%$ for maintenance in $100 \% \mathrm{O}_{2}$ ).

Tomographic images were reconstructed with a 3D filtered back projection (3D-FBP) algorithm ${ }^{35}$ using a 12th-order Butterworth filter at a $35 \%$ Nyquist frequency cut-off. The trans-axial and axial resolutions of the PET scanner were $1.65 \mathrm{~mm}$ and $1.9 \mathrm{~mm}$ full width at half-maximum (FWHM), respectively. The voxel size of the reconstructed images was isometric $\left(0.81 \mathrm{~mm}^{3}\right)$, the energy window was 400-700 keV, and decay, alignment, normalization, and deadtime corrections were applied.

\section{Magnetic Resonance Study}

An MRI study of one animal at PND100 was acquired with a 7-Tesla Biospec 70/20 scanner (Bruker, Ettlingen, Germany) using a rat head surface array coil centred on the brain. The animal was anesthetized with sevoflurane (4.5\% for induction and $2.5 \%$ for maintenance in $\left.100 \% \mathrm{O}_{2}\right)$ and placed on a stereotactic device to prevent movement during the acquisition. A T2-weighted spin echo sequence was acquired, with $\mathrm{TR}=4062 \mathrm{~ms}, \mathrm{TE}=33 \mathrm{~ms}$, flip angle $=90^{\circ}$, RARE factor $=8$. FOV $=3.7 \times 3.7 \mathrm{~cm}$, matrix size $=256 \times 256$, slice thickness $=0.8$ 
$\mathrm{mm}$ (37 slices). The inhomogeneity of magnetic field caused by the surface antenna was corrected.

This MRI study was only used as an anatomical template in order to display the results of the statistical analysis, since PET imaging has a lower anatomical resolution.

\section{Data Analysis}

\section{Morphine self-administration}

The statistical analysis of behavioral data consisted in a repeated-measures three-way ANOVA including the strain (LEW vs F344), treatment (morphine vs saline), and repetition time as factors. The average number of self-administered injections was also determined and analyzed using two-way ANOVA followed by a post hoc Bonferroni correction.

\section{PET: statistical parametric mapping analysis}

PET image post-processing and intensity normalization were performed following protocols previously described by our group 36,37 . Briefly, the reconstructed images were spatially registered using rigid transformations with an automatic algorithm based on mutual information ${ }^{38}$, thereby enabling voxelto-voxel comparisons. All PET data were smoothed with a 2-mm FWHM isotropic Gaussian kernel. A brain mask was manually segmented onto the MRI template and applied to the PET studies to remove extracerebral voxels and to ensure that only voxels mapping brain tissue were included in the analysis. Image intensity was normalized to the brain average value (100\%).

The statistical analysis was performed using SPM12 (Wellcome Department of Cognitive Neurology, London, UK) and consisted in the analysis of variance (ANOVA) of three fixed factors with repeated measures on one of them: strain (inter-subject factor; levels: LEW, F344), condition (inter-subject factor; levels: saline, morphine), and time (within-subject factor, levels: at the end of the MSA phase, at the end of the withdrawal phase). Results were considered significant at a threshold of $p<0.01$, uncorrected at the voxel level, but clusterbased-corrected by FWE in order to avoid a type II error. Only strain differences were significant for multiple comparisons using the family-wise error (FWE) rate with a significance level of $p<0.05$. A 10-voxel clustering (spatial-extent) threshold 
was also applied to reduce the possibility of a type I error; therefore, significant regions smaller than ten adjacent activated voxels were not admitted.

\section{RESULTS}

\section{Morphine self-administration}

The three-way ANOVA revealed a statistically significant effect of the strain $(p<0.01)$, treatment $(p<0.001)$, and time $(p<0.001)$. We also found an interaction for strain $\times$ treatment $(p<0.001)$ and time $\times$ treatment $(p<0.001)$. Thus, the number of morphine injections per session was greater for LEW animals than for F344 animals $(p<0.01)$ (Figure 1B). In addition, the average number of selfadministered injections over the 15 sessions was greater in the LEW-morphine (48.58 \pm 6.71$)$ group than in the LEW-saline $(9.90 \pm 3.32)$ and F344 rats (morphine: $15.36 \pm 4.40$; saline: $6.66 \pm 1.79$ ).

\section{PET: statistical parametric mapping analysis}

Table 1 shows the differences in brain glucose metabolism between rat strains, and the brain metabolic changes after the MSA study.

SPM analysis revealed a significant effect of the rat strain with higher metabolism in the hypothalamus and the cerebral cortex and lower FDG uptake in the brainstem (locus coeruleus) and PAG in the LEW animals than in the F344 animals (Figure 1C).

During the drug acquisition phase, F344-morphine animals showed higher glucose metabolism in the cortical area and lower FDG uptake in the motor and piriform cortex than the F344-saline animals (Figure 2A). LEW-morphine animals showed lower metabolism in the somatosensorial and cingulate cortex and the thalamus and higher FDG uptake in the cerebellum than the LEW-saline animals (Figure 2A).

During drug withdrawal, F344-morphine animals showed higher metabolism in the left cortex and lower FDG uptake in the restrosplenial and motor cortices than the F344-saline animals (Figure 2B). LEW-morphine animals showed higher metabolism in the cerebellum and piriform cortex and lower FDG uptake in the cortex, thalamus, hippocampus, and caudate putamen than the LEW-saline animals (Figure 2B). 


\section{DISCUSSION}

To our knowledge, this is the first report to show that brain glucose metabolism differs from strain to strain, and that this finding may account, among others, for the differences in morphine self-administration (MSA) between LEW and F344 animals.

One of the core traits shown by rat strains with differential vulnerability to drug addiction is the capacity to self-administer more drug than other rat strains 1,4,39,40. According to this, drug intake is very high in some vulnerable animals and very low in other resistant one ${ }^{40}$. Both inbred rat lines, LEW and F344, can learn to self-administer opioid drugs for extended periods, but even with equivalent exposure to the drugs, LEW rats are more likely to transition to addiction and are more likely to relapse than $\mathrm{F} 344$ rats ${ }^{9}$.

In this study, we found that LEW rats self-administered more morphine injections per session than F344 rats, as previously reported by others and also by our group ${ }^{9-11}$. Furthermore, the average number of self-administered injections over the 15 sessions was higher in LEW rats than in F344 rats, as previously shown ${ }^{9,10}$. Our data reinforce the fact that the LEW strain is a morphine-vulnerable phenotype predisposed to higher doses of morphine intake than the F344 strain.

The role of individual differences in the responses to drugs of abuse, and thus predisposition to addiction is not new, and it has been widely demonstrated in humans and laboratory animals ${ }^{4}$. An important issue in drug addiction is to understand whether a person is predisposed to addiction. Thus, differences in vulnerability to drug dependence may be associated with differences in the efficiency of neural substrates to translate the drug effects and, in turn, to differences in brain metabolism. In this respect, the development of PET scanners for laboratory animals enabling the in vivo study of the neurotoxic effects of drugs in a non-invasive follow-up provides an alternative way to explore the underlying mechanisms of morphine-induced neurotoxicity. In the present study, $\left[{ }^{18} \mathrm{~F}\right]-\mathrm{FDG}$ was used as a marker of cerebral glucose consumption, which indicates neuronal activity ${ }^{41}$. Our $\left[{ }^{18} \mathrm{~F}\right]-\mathrm{FDG}$ data showed that the LEW animals exhibited increased glucose metabolism in cortical areas, including the somatosensory and the entorhinal cortices and the hypothalamus compared with 
the F344 animals. Sensory system information plays an important role in responses related to drug addiction, and, specifically, the somatosensory system is required for the positive reward property of drugs ${ }^{42}$. In this sense, the higher level of glucose metabolism in this area in the LEW rats could indicate differences in sensory information between the strains.

In general, addiction involves pathological learning in the neural processes related to the reward system and, therefore, underlies long-term associative memory deficits ${ }^{43}$. Here, we demonstrated differences in glucose metabolism in the entorhinal cortex between LEW and F344 rats. This area is closely related to learning and memory and connects the hippocampal neocortex and the hypothalamus, which is the main source of afferents to the hippocampus ${ }^{44}$. Research has shown genetic differences between both strains in synaptic plasticity in the hippocampus ${ }^{45,46}$, which are translated into spatial learning and memory deficits. Thus, F344 animals are less effective at performing some behavioral tasks, such as the radial arm maze test and the Morris maze test ${ }^{47}$, in that they are more liable to errors and take longer to learn the task ${ }^{48}$. The increased metabolism in this area in the LEW strain, together with the fact that more morphine was self-administered than the F344 strain, might be related to the higher learning capacity shown in the LEW strain.

We also found higher metabolism levels in the hypothalamus (including the mammillary bodies, the VTA, and the medial forebrain bundle) in the LEW animals than in F344 animals. Of note, the mammillary bodies are also involved (with anterior and dorsomedial nuclei of the thalamus) in recognition memory ${ }^{49}$. Together with the increased metabolism in the cortical area, these results represents a plausible explanation for the greater learning capacity of the LEW strain. In addition, the hypothalamus is part of the hypothalamic-pituitary-adrenal (HPA) axis, and differences in HPA axis reactivity between LEW and F344 strains have been reported ${ }^{39}$. Therefore, the increased glucose metabolism in the hypothalamus in the LEW strain would support this alteration in the HPA axis, which is consistent with the alteration of the reward and motivational processes reported for these strains ${ }^{50}$.

In contrast, glucose metabolism was lower in the brainstem and PAG in the LEW strain than in the F344 strain. The brainstem controls basic vital 
functions, such as heart rate, breathing, and sleeping, but it is also involved in emotional responses and episodes of distress. In this respect, an increased state of anxiety in LEW rats has been associated with alteration of the HPA axis ${ }^{50}$. Furthermore, while the PAG is a key area in acute and chronic pain processing, it is also involved in mediating fear-evoked behavior, which is in turn related to anxiety and depression ${ }^{51}$. Taken together, these results could also account for the differences in HPA axis reactivity between the strains

Regarding the morphine self-administration study, during acquisition, morphine-F344 animals showed increased glucose metabolism in cortical areas, with more changes in the left hemisphere than in the right, and decreased FDG uptake in the motor and piriform cortices than saline-F344 animals. These changes were mainly maintained during drug withdrawal. However, the metabolism pattern of the morphine-LEW animals was completely different when compared to saline-LEW animals, with decreases in the somatosensorial cortex, thalamus, and cingulate cortex that were maintained and extended during withdrawal. The different brain metabolic patterns observed after the MSA study between these rat strains indicate differences in the efficiency of neural substrates to translate the drug effects and, in turn, possible differences in vulnerability to morphine abuse.

Few in vivo imaging studies have evaluated the effect of morphine or other opioids on glucose metabolism using PET or single photon emission computed tomography (SPECT) in humans, probably because of the radioactive nature of these techniques, being most of them from the 1990s and beginning of this century. London and coworkers showed that acute administration of morphine in humans reduces glucose uptake in the brain by $10 \%$ on average ${ }^{26}$, with more changes in the left hemisphere than in the right hemisphere. In our study, repeated exposure to morphine induced different patterns of brain changes depending on the rat strain. During acquisition phase, morphine resulted in reduced glucose metabolism in cortical areas in both strains. This pattern of cortical reduction increased after withdrawal in the LEW animals but not in the F344 animals. Repeated exposure to morphine is usually accompanied by the development of tolerance and dependence. In addition, although the exact mechanisms underlying these phenomena are not yet fully understood, they are 
known to be associated with drug-induced neuroadaptations ${ }^{43}$. In this respect, nuclear magnetic resonance spectroscopy studies demonstrated that acute administration of morphine produces a significant decrease in glycine and glutamate levels that were dramatically increased or overcompensated, when naloxone was used to precipitate withdrawal ${ }^{52}$. Thus, in our F344 animals, chronic morphine may have triggered some compensatory mechanisms in order to normalize glucose metabolism, which were not triggered in the LEW animals.

During drug withdrawal in the LEW strain, glucose metabolism decreased dramatically in brain regions associated with reward and drug dependence, such as the hippocampus, thalamus, caudate-putamen, and cingulate cortex; while glucose metabolism changes in the F344 strain were modest compared to the LEW strain. In humans, neurobiological abnormalities in the regional cerebral metabolic rate for glucose were found in chronic opiate users several years after methadone detoxification ${ }^{53}$. This widespread pattern of abnormal cortical activity involved the anterior cingulate cortex, left mid-cingulate cortex, left insula, and right superior frontal cortex ${ }^{53}$, similar to some of our cortical changes. In addition, SPECT studies have shown perfusion deficits during heroin withdrawal in several brain areas, including the temporal lobe ${ }^{54}$ and the frontal, parietal, and temporal areas in a chronic opioid user after one week of interrupted administration ${ }^{55}$. In our study, 15 days of morphine abstinence induced a pattern of abnormal brain activity in the LEW animals similar to that found in humans. However, this abnormal metabolic pattern was not found in the F344 animals, suggesting that the genetic background (and other factors) makes one individual more susceptible to developing morphine addiction than another. Furthermore, the LEW strain showed increased metabolism in the piriform cortex. This structure is an olfactory region, and its involvement in the behavioral effects of drugs is limited. Thus, the increased expression of the activity marker c-Fos in the piriform cortex has been associated with cocaine-induced conditioned place preference ${ }^{56}$ and has also been associated with relapse in opioid seeking after food choiceinduced voluntary abstinence ${ }^{57}$. Therefore, the increased metabolism in the piriform cortex in the LEW strain could respond to morphine seeking after the withdrawal period and could explain in part the implication of the piriform cortex in morphine addiction and dependence. 
Nonetheless, our study had several limitations. First, we only evaluated males. The effect of morphine on females could be different, so further studies would be advisable to determine how the gender may influence on the effect of morphine in brain metabolism. Second, we have not corrected for multiple comparisons since individual analysis methods (SPM) provide some correction, being a common practice in exploratory works ${ }^{58}$. In addition, Bonferroni correction assumes independence of the voxels, which is not true in brain imaging studies and would underestimate the real effects.

In conclusion, we found significant brain metabolic differences between LEW and F344 strains in brain regions associated with reward and drug dependence. In addition, the different brain metabolic patterns observed after the MSA study between these rat strains indicate differences in the efficiency of neural substrates to translate the drug effects, which could explain the differences in predisposition to morphine abuse between one individual and another. These findings have important implications for the use of these rat strains in translational morphine and opiate research.

\section{REFERENCES}

1 Reed, B. \& Kreek, M. J. Genetic Vulnerability to Opioid Addiction. Cold Spring Harbor perspectives in medicine, doi:10.1101/cshperspect.a039735 (2020).

2 Picciotto, M. R. \& Kenny, P. J. Mechanisms of Nicotine Addiction. Cold Spring Harbor perspectives in medicine, doi:10.1101/cshperspect.a039610 (2020).

3 Darcey, V. L. \& Serafine, K. M. Omega-3 Fatty Acids and Vulnerability to Addiction: Reviewing Preclinical and Clinical Evidence. Current pharmaceutical design 26, 2385-2401, doi:10.2174/1381612826666200429094158 (2020).

4 Jupp, B., Jones, J. A. \& Dalley, J. W. Modelling Differential Vulnerability to Substance Use Disorder in Rodents: Neurobiological Mechanisms. Handbook of experimental pharmacology 258, 203-230, doi:10.1007/164_2019_300 (2020).

5 O'Brien, C. P., Ehrman, R. N. \& Ternes, J. W. Classical conditioning in opiate dependence. NIDA Res Monogr 49, 35-46 (1984).

6 Li, T. K. \& Lumeng, L. Alcohol preference and voluntary alcohol intakes of inbred rat strains and the National Institutes of Health heterogeneous stock of rats. Alcoholism, clinical and experimental research 8, 485-486 (1984).

7 George, F. R. \& Goldberg, S. R. Genetic differences in responses to cocaine. NIDA Res Monogr 88, 239-249 (1988).

8 Suzuki, T., Otani, K., Koike, Y. \& Misawa, M. Genetic differences in preferences for morphine and codeine in Lewis and Fischer 344 inbred rat strains. Japanese journal of pharmacology 47, 425-431 (1988).

9 Garcia-Lecumberri, C. et al. Strain differences in the dose-response relationship for morphine self-administration and impulsive choice between Lewis and Fischer 344 rats. Journal of psychopharmacology 25, 783-791, doi:10.1177/0269881110367444 (2011). 
10 Sanchez-Cardoso, P. et al. Strain differences between Lewis and Fischer 344 rats in the modulation of dopaminergic receptors after morphine self-administration and during extinction. Neuropharmacology 57, 8-17, doi:10.1016/j.neuropharm.2009.03.014 (2009).

11 Sanchez-Cardoso, P. et al. Modulation of the endogenous opioid system after morphine self-administration and during its extinction: a study in Lewis and $\begin{array}{lllll}\text { Fischer } 344 \quad \text { rats. } & \text { Neuropharmacology }\end{array}$ doi:10.1016/j.neuropharm.2006.10.011 (2007).

12 Martin, S. et al. Genetic differences in NMDA and D1 receptor levels, and operant responding for food and morphine in Lewis and Fischer 344 rats. Brain research 973, 205-213 (2003).

13 Ucha, M. et al. Morphine self-administration alters the expression of translational machinery genes in the amygdala of male Lewis rats. Journal of psychopharmacology 33, 882-893, doi:10.1177/0269881119836206 (2019).

14 Guitart, X. et al. Lewis and Fischer rat strains display differences in biochemical, electrophysiological and behavioral parameters: studies in the nucleus accumbens and locus coeruleus of drug naive and morphine-treated animals. Brain research 611, 7-17 (1993).

15 Martin, S. et al. Differential basal proenkephalin gene expression in dorsal striatum and nucleus accumbens, and vulnerability to morphine selfadministration in Fischer 344 and Lewis rats. Brain research 821, 350-355 (1999).

16 Guitart, X., Beitner-Johnson, D., Marby, D. W., Kosten, T. A. \& Nestler, E. J. Fischer and Lewis rat strains differ in basal levels of neurofilament proteins and their regulation by chronic morphine in the mesolimbic dopamine system. Synapse 12, 242-253, doi:10.1002/syn.890120310 (1992).

17 Haile, C. N., Hiroi, N., Nestler, E. J. \& Kosten, T. A. Differential behavioral responses to cocaine are associated with dynamics of mesolimbic dopamine proteins in Lewis and Fischer 344 rats. Synapse 41, 179-190, doi:10.1002/syn.1073 (2001).

18 Kosten, T. A. \& Ambrosio, E. HPA axis function and drug addictive behaviors: insights from studies with Lewis and Fischer 344 inbred rats. Psychoneuroendocrinology 27, 35-69 (2002).

19 Parvaz, M. A., Alia-Klein, N., Woicik, P. A., Volkow, N. D. \& Goldstein, R. Z. Neuroimaging for drug addiction and related behaviors. Reviews in the neurosciences 22, 609-624, doi:10.1515/RNS.2011.055 (2011).

20 Volkow, N. D. et al. Reduced metabolism in brain "control networks" following cocaine-cues exposure in female cocaine abusers. PloS one 6, e16573, doi:10.1371/journal.pone.0016573 (2011).

21 Volkow, N. D. et al. Changes in brain glucose metabolism in cocaine dependence and withdrawal. The American journal of psychiatry 148, 621-626, doi:10.1176/ajp.148.5.621 (1991).

22 Volkow, N. D. et al. Acute alcohol intoxication decreases glucose metabolism but increases acetate uptake in the human brain. NeuroImage 64, 277-283, doi:10.1016/j.neuroimage.2012.08.057 (2013).

23 Domino, E. F., Tsukada, H. \& Harada, N. Positron emission tomographic measure of brain dopamine dependence to nicotine as a model of drugs of abuse. Psychopharmacology 204, 149-153, doi:10.1007/s00213-008-1445-8 (2009).

24 Volkow, N. D. et al. Decreased dopamine brain reactivity in marijuana abusers is associated with negative emotionality and addiction severity. Proceedings of the 
National Academy of Sciences of the United States of America 111, E3149-3156, doi:10.1073/pnas.1411228111 (2014).

25 Auvity, S. et al. Acute Morphine Exposure Increases the Brain Distribution of [18F]DPA-714, a PET Biomarker of Glial Activation in Nonhuman Primates. The international journal of neuropsychopharmacology 20, 67-71, doi:10.1093/ijnp/pyw077 (2017).

26 London, E. D. et al. Morphine-induced metabolic changes in human brain. Studies with positron emission tomography and [fluorine 18]fluorodeoxyglucose. Archives of general psychiatry 47, 73-81, doi:10.1001/archpsyc.1990.01810130075010 (1990).

27 Chen, Q. et al. PET Imaging Reveals Brain Metabolic Changes in Adolescent Rats Following Chronic Escalating Morphine Administration. Molecular imaging and biology 20, 993-1000, doi:10.1007/s11307-018-1188-9 (2018).

28 Gatley, S. J. et al. PET imaging in clinical drug abuse research. Current pharmaceutical design 11, 3203-3219, doi:10.2174/138161205774424717 (2005).

29 Soto-Montenegro, M. L. et al. Effects of MDMA on blood glucose levels and brain glucose metabolism. Eur J Nucl Med Mol Imaging 34, 916-925, doi:10.1007/s00259-006-0262-8 (2007).

30 Soto-Montenegro, M. L. et al. Functional neuroimaging of amphetamine-induced striatal neurotoxicity in the pleiotrophin knockout mouse model. Neurosci Lett 591, 132-137, doi:10.1016/j.neulet.2015.02.041

S0304-3940(15)00155-X [pii] (2015).

31 Burns, J. A. et al. Molecular Imaging of Opioid and Dopamine Systems: Insights Into the Pharmacogenetics of Opioid Use Disorders. Frontiers in psychiatry 10, 626, doi:10.3389/fpsyt.2019.00626 (2019).

32 Fowler, J. S., Volkow, N. D., Kassed, C. A. \& Chang, L. Imaging the addicted human brain. Science \& practice perspectives 3, 4-16, doi:10.1151/spp07324 (2007).

33 Volkow, N. D., Fowler, J. S., Wang, G. J., Baler, R. \& Telang, F. Imaging dopamine's role in drug abuse and addiction. Neuropharmacology 56 Suppl 1, 38, doi:10.1016/j.neuropharm.2008.05.022 (2009).

34 Wiers, C. E., Cabrera, E., Skarda, E., Volkow, N. D. \& Wang, G. J. PET imaging for addiction medicine: From neural mechanisms to clinical considerations. Progress in brain research 224, 175-201, doi:10.1016/bs.pbr.2015.07.016 (2016).

35 Abella, M., Vaquero, J. J., Soto-Montenegro, M. L., Lage, E. \& Desco, M. Sinogram bow-tie filtering in FBP PET reconstruction. Medical physics 36, 16631671, doi:10.1118/1.3096707 (2009).

36 Hadar, R. et al. Using a maternal immune stimulation model of schizophrenia to study behavioral and neurobiological alterations over the developmental course. Schizophrenia research 166, 238-247, doi:10.1016/j.schres.2015.05.010 (2015).

37 Soto-Montenegro, M. L., Pascau, J. \& Desco, M. Response to deep brain stimulation in the lateral hypothalamic area in a rat model of obesity: in vivo assessment of brain glucose metabolism. Mol Imaging Biol 16, 830-837, doi:10.1007/s11307-014-0753-0 (2014).

38 Pascau, J. et al. Automated method for small-animal PET image registration with intrinsic validation. Mol Imaging Biol 11, 107-113 (2009). 
39 Cadoni, C. Fischer 344 and Lewis Rat Strains as a Model of Genetic Vulnerability to Drug Addiction. Frontiers in neuroscience 10, 13, doi:10.3389/fnins.2016.00013 (2016).

40 Piazza, P. V., Deroche-Gamonent, V., Rouge-Pont, F. \& Le Moal, M. Vertical shifts in self-administration dose-response functions predict a drug-vulnerable phenotype predisposed to addiction. The Journal of neuroscience : the official journal of the Society for Neuroscience 20, 4226-4232 (2000).

41 Shimoji, K. et al. Measurement of cerebral glucose metabolic rates in the anesthetized rat by dynamic scanning with 18F-FDG, the ATLAS small animal PET scanner, and arterial blood sampling. Journal of nuclear medicine : official publication, Society of Nuclear Medicine 45, 665-672 (2004).

42 Meng, Z., Liu, C., Hu, X. \& Ma, Y. Somatosensory cortices are required for the acquisition of morphine-induced conditioned place preference. PloS one 4, e7742, doi:10.1371/journal.pone.0007742 (2009).

43 Nestler, E. J. Molecular mechanisms of drug addiction. Neuropharmacology 47 Suppl 1, 24-32, doi:10.1016/j.neuropharm.2004.06.031 (2004).

44 Kandel., E., Schwartz., J. \& Jessel., T. Principles of Neural Science. 4th edn, (McGraw-Hill, 2000).

45 Miguens, M. et al. Depotentiation of hippocampal long-term potentiation depends on genetic background and is modulated by cocaine self-administration. Neuroscience 187, 36-42, doi:10.1016/j.neuroscience.2011.04.056 (2011).

46 Prakash, S., Ambrosio, E., Alguacil, L. F. \& Del Olmo, N. Genetic differences in hippocampal synaptic plasticity. Neuroscience 161, 342-346, doi:10.1016/j.neuroscience.2009.03.061 (2009).

47 van der Staay, F. J., Arndt, S. S. \& Nordquist, R. E. Evaluation of animal models of neurobehavioral disorders. Behavioral and brain functions : BBF 5, 11, doi:10.1186/1744-9081-5-11 (2009).

48 Fole, A. et al. Effects of chronic cocaine administration on spatial learning and hippocampal spine density in two genetically different strains of rats. Neurobiology of learning and memory 95, 491-497, doi:10.1016/j.nlm.2011.02.012 (2011).

49 Aggleton, J. P. et al. Hippocampal-anterior thalamic pathways for memory: uncovering a network of direct and indirect actions. The European journal of neuroscience 31, 2292-2307, doi:10.1111/j.1460-9568.2010.07251.x (2010).

50 Cohen, H. et al. Blunted HPA axis response to stress influences susceptibility to posttraumatic stress response in rats. Biological psychiatry 59, 1208-1218, doi:10.1016/j.biopsych.2005.12.003 (2006).

51 Mokhtar, M. \& Singh, P. in StatPearls (2020).

52 Sharma, S. K. et al. Alterations in brain metabolism induced by chronic morphine treatment: NMR studies in rat CNS. Neurochemical research 28, 1369-1373 (2003).

53 Galynker, II et al. Cerebral metabolism and mood in remitted opiate dependence. Drug and alcohol dependence 90, 166-174, doi:10.1016/j.drugalcdep.2007.03.015 (2007).

54 Danos, P. et al. Pathological regional cerebral blood flow in opiate-dependent patients during withdrawal: a HMPAO-SPECT study. Neuropsychobiology 37, 194-199, doi:10.1159/000026502 (1998).

55 Rose, J. S. et al. Cerebral perfusion in early and late opiate withdrawal: a technetium-99m-HMPAO SPECT study. Psychiatry research 67, 39-47 (1996). 
56 Crawford, C. A., McDougall, S. A., Bolanos, C. A., Hall, S. \& Berger, S. P. The effects of the kappa agonist U-50,488 on cocaine-induced conditioned and unconditioned behaviors and Fos immunoreactivity. Psychopharmacology 120, 392-399, doi:10.1007/BF02245810 (1995).

57 Reiner, D. J. et al. Role of Projections between Piriform Cortex and Orbitofrontal Cortex in Relapse to Fentanyl Seeking after Palatable Food Choice-Induced Voluntary Abstinence. The Journal of neuroscience : the official journal of the Society for Neuroscience 40, 2485-2497, doi:10.1523/JNEUROSCI.269319.2020 (2020).

58 Althouse, A. D. Adjust for Multiple Comparisons? It's Not That Simple. The Annals of thoracic surgery 101, 1644-1645, doi:10.1016/j.athoracsur.2015.11.024 (2016).

\section{ACKNOWLEDGEMENTS}

Morphine sulphate was kindly provided by the Dirección General de Estupefacientes (Spain).

\section{ROLE OF FUNDING SOURCE}

MLS was supported by the Ministerio de Ciencia, Innovación y Universidades, Instituto de Salud Carlos III (projects PI14/00860 and PI17/01766, and grant CPII14/00005), co-funded by the European Regional Development Fund (ERDF), "A way of making Europe", CIBERSAM, Delegación del Gobierno para el Plan Nacional sobre Drogas (2017/085), Fundación Mapfre, and Fundación Alicia Koplowitz. EA was supported by grants from Ministerio de Sanidad, Servicios Sociales e Igualdad and Red de Trastornos Adictivos del Instituto de Salud Carlos III (RTA-RD16/020/0022), Delegación del Gobierno para el Plan Nacional sobre Drogas (2016I073), Ministerio de Ciencia e Innovación (PSI201680541-P), UNED (Plan de Promoción de la Investigación 2018-2020), and the European Union's Justice Programme - Drugs Policy Initiatives (JUST-2017-AGDRUGS- 806996-JUSTSO). NLR was supported by Instituto de Investigación Sanitaria Gregorio Marañón, "Programa Intramural de Impulso a la I+D+I 2019". The CNIC is supported by the Instituto de Salud Carlos III (ISCIII), the Ministerio de Ciencia e Innovación (MCIN), and the Pro CNIC Foundation, and is a Severo Ochoa Center of Excellence (SEV-2015-0505).

\section{AUTHOR CONTRIBUTIONS}

MLS: Acquisition and analysis of imaging studies, writing-original draft. VG: Analysis of imaging studies. NLR: Analysis of behavioral studies, writing-original draft. GLM: Animal handle, review \& editing. MD: Review \& editing. EA: 
Conception of the study, animal handle, review \& editing. All authors reviewed and approved the final version of the manuscript to be published.

\section{COMPLIANCE WITH ETHICAL STANDARDS}

Competing interests: The authors declare no competing interests.

Ethical approval: All applicable international, national, and/or institutional guidelines for the care and use of animals were followed.

\section{FIGURE LEGENDS}

Figure 1. Study design, behavioral study and differences in brain glucose metabolism between rat strains. A) Representative diagram of the chronology of the experimental procedures performed during the study according to the age of the animals. Abbrev.: CT, computerized tomography; GD, gestational day; 18FDG, [18F]Fluorodeoxyglucose; IOS, inflammatory/oxidative markers, PET, positron emission tomography; PND, postnatal day. B) MSA study in LEW and F344 rats. Morphine (1 $\mathrm{mg} / \mathrm{kg}$ ) or saline self-administration in adult LEW and F344 rats under an FR1 schedule of reinforcement. The values are expressed as the mean \pm SEM: LEW-saline $(n=6)$, LEW-morphine $(n=8)$, F344-saline $(n=6)$, and F344-morphine $(n=7)$. The number of morphine injections per session was greater for LEW animals than for F344 animals. 3way ANOVA followed by Bonferroni's multiple comparisons test $\left[{ }^{*} p<0.5\right.$ and ${ }^{* *} p<0.01$ vs saline animals]. C) Colored PET overlays on the MRI reference indicate increased FDG uptake (hot colors) or decreased FDG uptake (cold colors) in LEW animals compared with F344 animals. Statistics corrected for multiple comparisons (FWE, $\mathrm{p}<0.05$ ). Region of interest (BS: brainstem, Cb: Cerebellum, Hypoth: hypothalamus, PAG: periaqueductal gray matter). Side: left (L) and right (R). $k$ : cluster size, T: Student $t$. Glucose metabolism: increase ( $\uparrow)$ and decrease ( $\downarrow$ ). $p$ : $p$ value (unc: uncorrected, FWE: family-wise error).

Figure 2. Brain glucose metabolism patterns associated with morphine consumption in F344 and LEW rat strains. Colored PET overlays on the MRI reference indicate increased FDG uptake (hot colors) or decreased FDG uptake (cold colors) in the F344 (Left) and LEW (Right) strains after the acquisition (A) or the withdrawal $(B)$ phases compared with the saline-treated: LEW-saline $(n=5)$, LEWmorphine $(n=6)$, F344-saline $(n=7)$, and F344-morphine $(n=6)$. Threshold for statistical significance of $\mathrm{p}<0.01$. Region of interest (C: cortex, CC: cingulate cortex, $\mathrm{Cb}$ : Cerebellum, CP: caudate putamen, Hipp: hippocampus, MC: motor cortex, PirC: piriform cortex, RSC: retrosplenial cortex, Sept: septum, SSC: somatosensorial cortex; Th: thalamus). Side: left $(L)$ and right (R). $k$ : cluster size, $T$ : Student $t$. Glucose metabolism: increase ( $\uparrow$ ) and decrease ( $\downarrow)$. $p: p$ value (unc: uncorrected, FWE: family-wise error). 
1. Brain metabolic changes after the MSA study

\begin{tabular}{|c|c|c|c|c|c|c|c|c|c|c|c|c|c|c|c|}
\hline & \multicolumn{7}{|c|}{ A) ACQUISITION PHASE } & \multicolumn{7}{|c|}{ B) WITHDRAWAL PHASE } \\
\hline & & ROI & Side & $\mathrm{t}$-value & $\begin{array}{c}\text { Punc } \\
\text { peak }\end{array}$ & $\begin{array}{l}\begin{array}{l}\text { p } \\
\text { FWE } \\
\text { peak }\end{array} \\
\end{array}$ & $\begin{array}{c}\begin{array}{c}\text { PFWE } \\
\text { cluster }\end{array} \\
\end{array}$ & $\mathrm{K}$ & ROI & Side & $\mathrm{t}$-value & $\begin{array}{c}\text { Punc } \\
\text { peak }\end{array}$ & $\begin{array}{l}\begin{array}{c}\text { PFDR } \\
\text { peak }\end{array} \\
\end{array}$ & $\begin{array}{c}p_{\text {FDR }} \\
\text { cluster }\end{array}$ & $\mathrm{K}$ \\
\hline \multirow{5}{*}{ 怘 } & \multirow{2}{*}{$\uparrow$} & \multirow{2}{*}{ Cortex } & $\mathrm{R}$ & 4.42 & \multirow{2}{*}{$<0.001$} & 0.128 & 0.053 & 129 & Cortex & L & 4.28 & \multirow{2}{*}{$<0.001$} & 0.160 & \multirow{2}{*}{$\begin{array}{l}0.197 \\
0.254\end{array}$} & \multirow{2}{*}{$\begin{array}{l}67 \\
56\end{array}$} \\
\hline & & & L & 13.08 & & 0.145 & 0.248 & 57 & $\mathrm{Cb}$ & -- & 4.05 & & 0.230 & & \\
\hline & \multirow{3}{*}{$\downarrow$} & MC & -- & 4.66 & \multirow{2}{*}{$<0.001$} & 0.085 & 0.216 & 63 & \multirow{3}{*}{ RSC-MC } & \multirow{3}{*}{--} & \multirow{3}{*}{5.00} & \multirow{3}{*}{$<0.001$} & \multirow{3}{*}{0.046} & \multirow{3}{*}{0.055} & \multirow{3}{*}{127} \\
\hline & & \multirow{2}{*}{ Pir C } & $\mathrm{R}$ & 4.03 & & 0.237 & 0.700 & 13 & & & & & & & \\
\hline & & & $\mathrm{L}$ & 3.62 & 0.001 & 0.421 & 0.314 & 47 & & & & & & & \\
\hline \multirow{5}{*}{ 疍 } & \multirow{3}{*}{$\uparrow$} & \multirow{3}{*}{$\mathrm{Cb}$} & \multirow{3}{*}{--} & \multirow{3}{*}{3.57} & \multirow{3}{*}{0.001} & \multirow{3}{*}{0.452} & \multirow{3}{*}{0.272} & \multirow{3}{*}{53} & $\mathrm{Cb}$ & & 3.54 & 0.001 & 0.467 & 0.079 & 109 \\
\hline & & & & & & & & & \multirow{2}{*}{ Pir C } & $\mathrm{R}$ & 5.32 & $<0.001$ & 0.026 & 0.361 & 41 \\
\hline & & & & & & & & & & $\mathrm{L}$ & 3.70 & 0.001 & 0.380 & 0.505 & 27 \\
\hline & \multirow{2}{*}{$\downarrow$} & \multirow{2}{*}{ SSC-Th-CC } & \multirow{2}{*}{ L } & \multirow{2}{*}{4.37} & \multirow{2}{*}{$<0.001$} & \multirow{2}{*}{0.137} & 0078 & 14 & C Th H ling & $\mathrm{R}$ & 4.63 & 0001 & 0.089 & 0001 & 00001 \\
\hline & & & & & & & $0.0 / 6$ & 1711 & C- & $\mathrm{L}$ & 5.09 & $<0.001$ & 0.009 & $<0.001$ & $<0.001$ \\
\hline
\end{tabular}

\section{Differences in brain glucose metabolism between rat strains}

\begin{tabular}{|c|c|c|c|c|c|c|c|c|c|c|c|c|c|c|c|}
\hline & ROI & Side & $\mathrm{t}$-value & $\begin{array}{c}\text { Punc }_{\text {unc }} \\
\text { peak }\end{array}$ & $\begin{array}{l}\text { p }_{\text {FWE }} \\
\text { peak }\end{array}$ & $\begin{array}{c}P_{\text {FWE }} \\
\text { cluster }\end{array}$ & K & & ROI & Side & t-value & $\begin{array}{c}\text { Punc } \\
\text { peak }\end{array}$ & $\begin{array}{l}\text { p }_{\text {FWE }} \\
\text { peak }\end{array}$ & $\begin{array}{l}P_{\text {FWE }} \\
\text { cluster }\end{array}$ & $\mathrm{K}$ \\
\hline \multirow[t]{2}{*}{$\uparrow$} & Hypoth & $\bar{R}$ & 13.08 & $<0.001$ & $<0.001$ & $<0.001$ & \multirow[t]{2}{*}{420} & \multirow[t]{2}{*}{$\downarrow$} & & -- & 9.72 & $<0.001$ & $<0.001$ & $<0.001$ & \multirow[t]{2}{*}{257} \\
\hline & & L & 9.72 & $<0.001$ & $<0.001$ & $<0.001$ & & & PAG & -- & 7.49 & $<0.001$ & $<0.001$ & $<0.001$ & \\
\hline
\end{tabular}

Table 1. Brain metabolic changes after the MSA study (1) and differences in brain glucose metabolism between rat strains (2). Type of change ( $\uparrow$ increase, $\downarrow$ decrease), ROI: region of interest, Side (L: left, R: right), t-value (T), statistical p-value (p) (uncorrected and corrected for multiple comparisons, FWE) at peak and cluster level, $\mathrm{k}$ (cluster size in number of voxels). Abbrev.: BS: brainstem; C: cortex; Cb: cerebellum; CC: cingulate cortex; CP: caudate-putamen; Ent C: entorhinal cortex; Hipp: hippocampus; Hypoth: hypothalamus; PAG: periaqueductal gray matter; Pir C: piriform cortex; RSC: retrosplenial cortex; SSC: somatosensorial cortex; Th: thalamus 


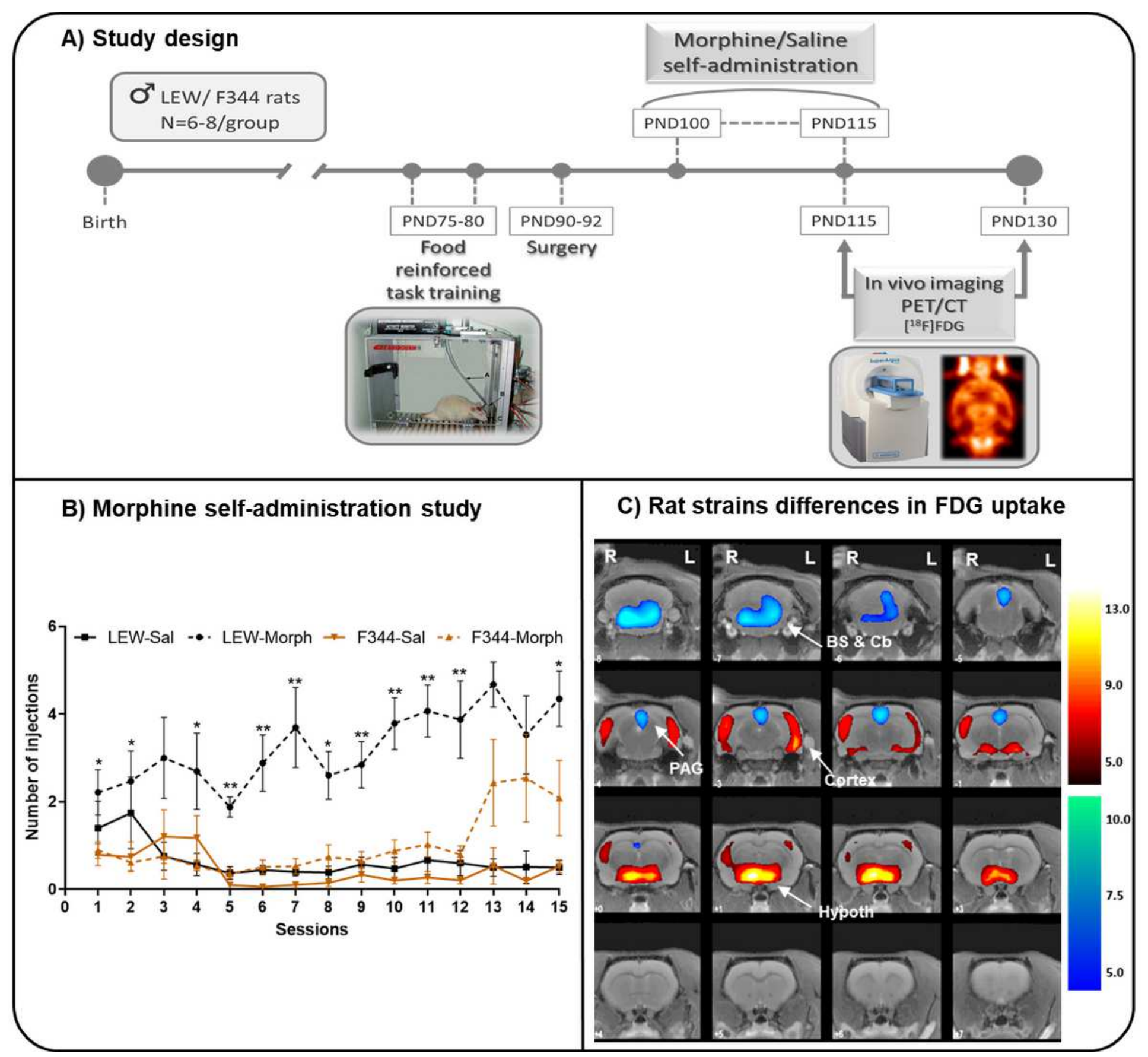

Figure 1

Study design, behavioral study and differences in brain glucose metabolism between rat strains. A) Representative diagram of the chronology of the experimental procedures performed during the study according to the age of the animals. Abbrev.: CT, computerized tomography; GD, gestational day; 18FDG, [18F]- Fluorodeoxyglucose; IOS, inflammatory/oxidative markers, PET, positron emission tomography; PND, postnatal day. B) MSA study in LEW and F344 rats. Morphine $(1 \mathrm{mg} / \mathrm{kg})$ or saline selfadministration in adult LEW and F344 rats under an FR1 schedule of reinforcement. The values are expressed as the mean \pm SEM: LEW-saline $(n=6)$, LEW-morphine $(n=8)$, F344-saline $(n=6)$, and F344- 
morphine $(n=7)$. The number of morphine injections per session was greater for $L E W$ animals than for F344 animals. 3- way ANOVA followed by Bonferroni's multiple comparisons test [ ${ }^{*} p<0.5$ and ${ }^{* *} p<0.01$ vs saline animals]. C) Colored PET overlays on the MRI reference indicate increased FDG uptake (hot colors) or decreased FDG uptake (cold colors) in LEW animals compared with F344 animals. Statistics corrected for multiple comparisons (FWE, $p<0.05$ ). Region of interest (BS: brainstem, Cb: Cerebellum, Hypoth: hypothalamus, PAG: periaqueductal gray matter). Side: left (L) and right (R). k: cluster size, $\mathrm{T}$ : Student t. Glucose metabolism: increase ( $(\mathbb{)})$ and decrease ( $(\mathbb{)})$. p: p value (unc: uncorrected, FWE: family-wise error).

\section{A) Acquisition phase}

F344

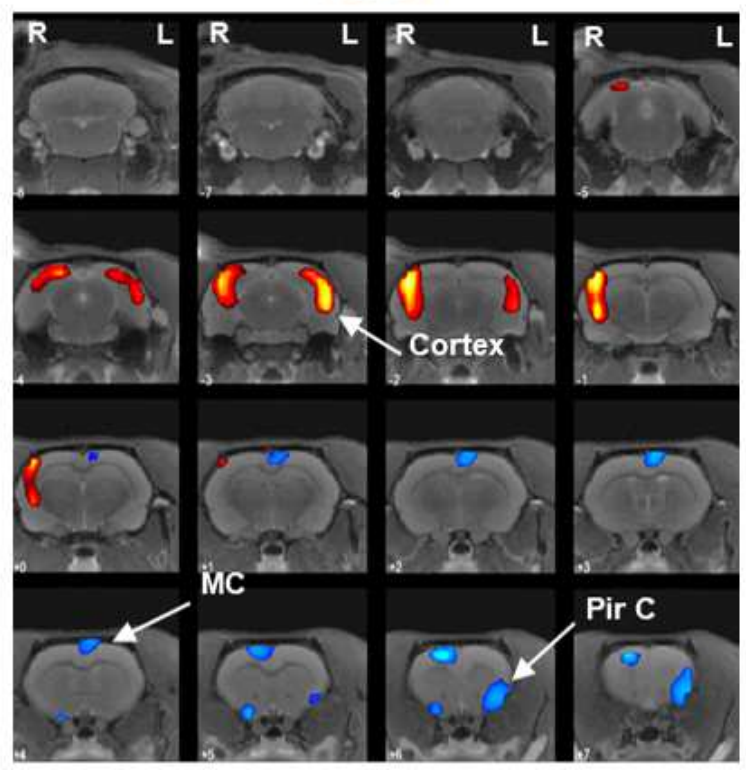

LEW
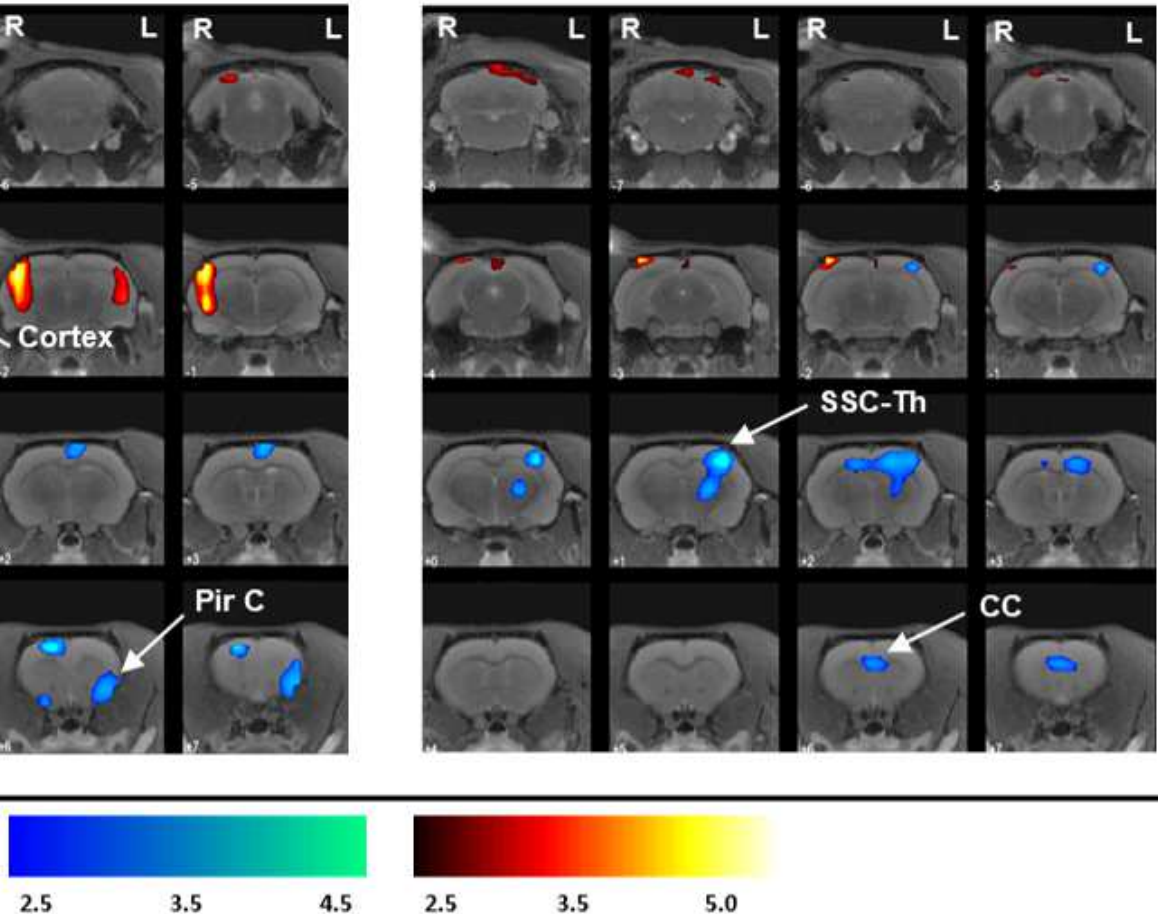

\section{B) Withdrawal phase}

F344

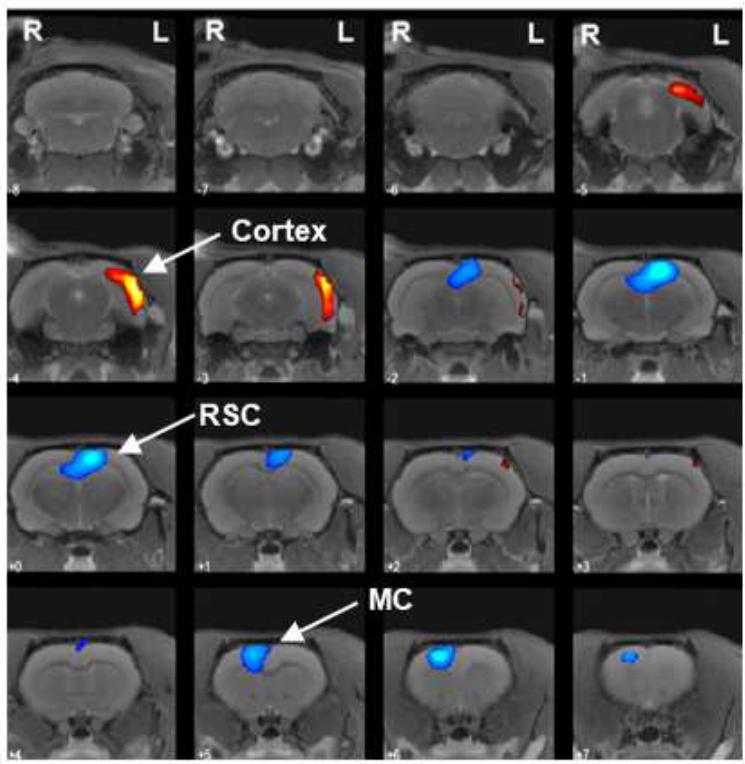

LEW

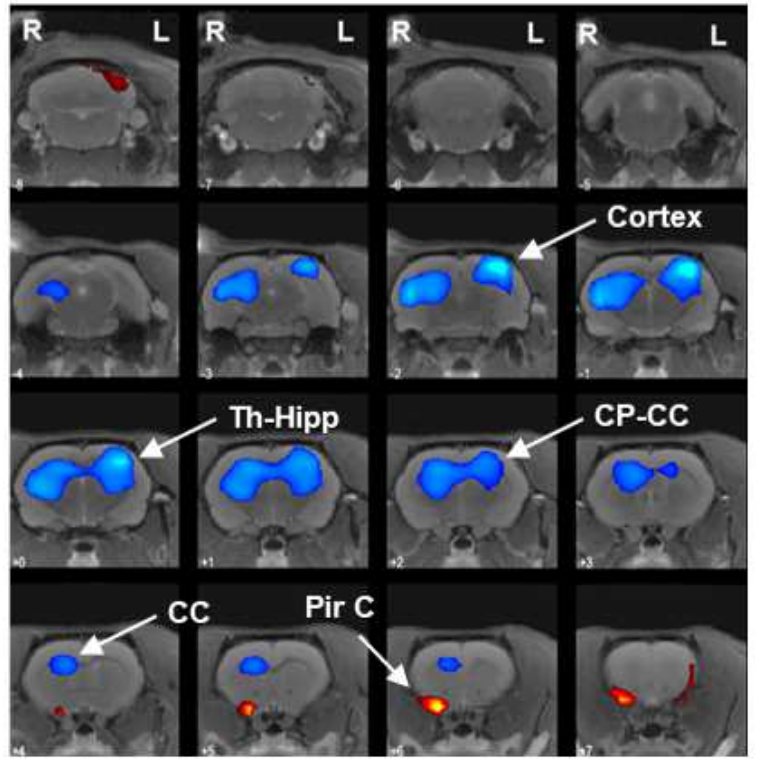




\section{Figure 2}

Brain glucose metabolism patterns associated with morphine consumption in F344 and LEW rat strains. Colored PET overlays on the MRI reference indicate increased FDG uptake (hot colors) or decreased FDG uptake (cold colors) in the F344 (Left) and LEW (Right) strains after the acquisition (A) or the withdrawal (B) phases compared with the saline-treated: LEW-saline $(n=5)$, LEW $7)$, and F344-morphine $(n=6)$. Threshold for statistical significance of $p<0.01$. Region of interest (C: cortex, CC: cingulate cortex, Cb: Cerebellum, CP: caudate putamen, Hipp: hippocampus, MC: motor cortex, PirC: piriform cortex, RSC: retrosplenial cortex, Sept: septum, SSC: somatosensorial cortex; Th: thalamus).

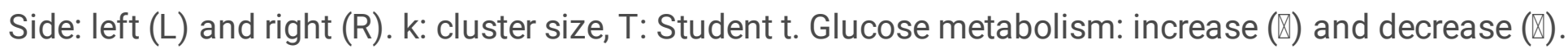
$p: p$ value (unc: uncorrected, FWE: family-wise error) 